\title{
Ajuste de la violencia familiar peruana a la teoría psicobiogeográfica de la salud mental ${ }^{1}$
}

\author{
Federico R. León ${ }^{2}$ \\ León \& Bustamante Consultores, Perú
}

Considerando el grado de exposición a radiación ultravioleta, la teoría psicobíogeográfica predice una variación latitudinal de la salud mental. Respuestas de 12604 mujeres en la Encuesta Demográfica y de Salud Familiar Perú 2000 fueron analizadas para determinar si la violencia física y psicológica ejercida por esposos y padres aumenta con la distancia al ecuador. Los efectos de la latitud fueron consistentes con la teoría en el desierto del Pacífico, la estepa serrana, la puna, y la eco-región Yunga, no así en la Amazonía, tal vez por la producción excesiva de vitamina $\mathrm{D}$ al norte de 50 50' S. No se halló efectos de la altura, pero los de la urbanización y la corriente de Humboldt fueron los predichos.

Palabras clave: latitud, radiación ultravioleta, violencia familiar.

\section{Adjustment of Peruvian family violence to the psychobiogeographic theory of mental health}

The psychobiogeographic theory, considering the extent of exposure to ultraviolet radiation, predicts a latitudinal variation of mental health. An analysis of questionnaire responses from 12,604 women in the Peru 2000 Demographic and Family Health Survey was undertaken to test the hypothesis that physical and psychological violence exerted by husbands and parents against women increases with distance from the Equator. The observed effects of latitude were consistent with the theory in the Pacific desert, Sierra steppe, Puna, and Yunga sites, but not in the Amazon eco-region, which could be due to an excess of vitamin $\mathrm{D}$ produced north of $5^{\circ} 50^{\prime} \mathrm{S}$. The theory's hypotheses concerning urbanization and the Humboldt Current were supported, but the one on altitude was not.

Keywords: latitude, ultraviolet radiation, family violence.

$1 \quad$ Nina Bustamante de León proporcionó los fondos para esta investigación y útiles sugerencias. Estoy agradecido a mi esposa y socia, así como a Demographic and Health Surveys por su permiso para analizar datos de la ENDES Perú 2000.

2 Socio, León \& Bustamante Consultores (www.leonybustamante.com). Dirección postal: Av. Javier Prado 7427, Lima 3. Correo electrónico: federicorleone@gmail.com. 

La teoría psicobíogeográfica (PBG) del deseo de hijos, poder doméstico y salud mental (León, 2012a) parte del reconocimiento de una verdad empírica universalmente aceptada: la latitud está estrechamente relacionada a ciertos factores climáticos. Específicamente, la radiación ultravioleta (RUV) decae desde la línea ecuatorial hacia los polos norte $(\mathrm{N})$ y sur $(\mathrm{S})$ por efecto del eje de rotación del planeta. El día 29 de mayo del 2012, por ejemplo, RUV alcanzó 13.4 en Quito, 8.7 en Lima, 8.8 en La Paz, 4.7 en Asunción, y 2.8 en Buenos Aires (TuTiempo. net, 2012). El que en Lima no haya alcanzado 10 o más es atribuible al efecto de filtro de la bruma causada por la fría corriente marina de Humboldt, un factor ya incorporado a la teoría (León, 2012b).

La RUV es relevante a la sexualidad, pese a que un reporte antiguo puso en duda el que las noches más largas y la variación estacional mayor que se observan con la cercanía a los polos tuvieran efectos sobre la testosterona (Huhtaniemi, Martikainen \& Tapanainen, 1982). Estudios más recientes demuestran en el hemisferio $\mathrm{N}$ que la exposición a la luz solar tiene un impacto en la producción de testosterona y estrógeno; los niveles en plasma declinan de noviembre a abril, es decir, durante el invierno, y comienzan a incrementarse continuamente durante la primavera y el verano (van Anders, Hampson \& Watson, 2006). Ello influye directamente en la tasa de concepciones humanas, que correlaciona positivamente con la intensidad de luz solar al momento de la concepción (Cummings, 2007). Debe quedar poca duda de que es RUV y no los rayos infrarrojos, causantes de la temperatura en superficie, lo que vincula a la latitud con las hormonas sexuales. RUV ocasiona cambios estructurales en las moléculas que se requieren en ciertas reacciones bioquímicas de la piel y retina.

La intensidad y duración de la exposición a RUV es proporcional a la tasa de síntesis de estas moléculas para producir vitamina $D$; de hecho, RUV constituye largamente la principal fuente de esta vitamina 
en el cuerpo humano. La vitamina D influye en las hormonas sexuales mediante su rol regulador en la síntesis del estrógeno en las gónadas de machos y hembras, como se ha demostrado en roedores; parece que esta acción se explica parcialmente por el mantenimiento de la homeostasis del calcio (Kinuta, Tanaka, Moriwake, Aya, Kato \& Seino, 2000). También se ha demostrado el rol de la vitamina $\mathrm{D}$ en la producción de testosterona humana; las dos variables están estrechamente correlacionadas y varían estacionalmente al unísono (Wehr, Pitz, Boehm, März \& ObermayerPietsch, 2009). RUV y la vitamina D, entonces, predicen una relación entre latitud y sexualidad. El creciente deseo de hijos de la mujer peruana al aproximarse al ecuador que se encontró y confirmó en diferentes muestras nacionales de gran envergadura (León, 1984, 1986, 2012a; Morales, 1983) puede, pues, explicarse por el aumento en la producción de vitamina $\mathrm{D}$ y sus efectos en los niveles de estrógeno. Ello también explicaría el mayor número de cerámicos precolombinos de contenido sexual explícito en el N que el S del Perú descubierto por León (1986) en las colecciones arqueológicas examinadas por Kauffmann-Doig (1979).

Como quiera que las hormonas sexuales hacen más masculino y dominante al hombre y más femenina y tímida a la mujer (McLean \& Anderson, 2009; véase también Costa, Terracciano \& McCrae, 2001), debe esperarse que las parejas sean más asimétricas en poder cerca al ecuador y más igualitarias con la distancia de la línea ecuatorial. Hallazgos recientes en muestras peruanas a gran escala (León 2011ab, 2012a) son consistentes con esta predicción. La correlación negativa entre deseo de hijos y poder doméstico de la mujer que se observó en el $\mathrm{N}$ del Perú se puede entender en función de la ocurrencia de máxima diferenciación sexual con la proximidad a la latitud $0^{\circ} 0^{\prime}$; al aumentar la distancia de la línea ecuatorial y decaer la diferenciación sexual, desaparece la correlación negativa entre deseo de hijos y poder doméstico de la mujer (León, 2012a).

La teoría PBG ofrece una red nomológica en la cual situar a la hipótesis de León (2011b) sobre la intermediación de un rasgo de personalidad entre la latitud y el poder doméstico de la mujer. Hay que tener en cuenta que este poder proviene de diversas fuentes. Por un lado están las normas sociales que amplían o restringen el rango de conductas 
permisibles según el género de la persona (Kabeer, 2001; Mason, 1998; Sen, 1999). La literatura también muestra que la edad, el nivel educativo y el trabajo por dinero de la mujer fortalecen su poder doméstico, y que la diferencia de edad con la del marido debilita sus decisiones autónomas (Kishor \& Subaiya, 2008). Habiendo sido estas variables mantenidas constantes en las regresiones - con excepción de la edad, que no correlacionó consistentemente con la latitud-, León (2011b) concluyó que el control económico del hogar por la mujer que halló relacionado con la meridionalidad tiene una fuente distinta de poder.

El poder doméstico residual de la mujer, es decir, no explicado por las otras variables, provendría de la asertividad, un aspecto de la extraversión (Eysenck \& Eysenck, 1985; Goldberg, 1973) que varía importantemente según el género (Costa, Terracciano \& McCrae, 2001). De acuerdo a la teoría PBG, el nivel de asertividad de la mujer crecería de $\mathrm{N}$ a $\mathrm{S}$ en el hemisferio $\mathrm{S}$ y de $\mathrm{S}$ a $\mathrm{N}$ en el hemisferio $\mathrm{N}$ por efecto de la relación causal que involucra a la intensidad de RUV, el nivel de vitamina D, y la diferenciación sexual (ver Figura 1). Los trabajos de Bem (1974), Wiggins (1979) y Costa y McCrae (1992) son consistentes con la teoría al oponer la asertividad (asociada supuestamente al poder doméstico, que crece de $\mathrm{N}$ a $\mathrm{S}$ ) al amor (asociado supuestamente al deseo de hijos, que crece de $S$ a N).

La teoría PBG de la salud mental (León, 2012a) fue formulada como una extensión de la del deseo de hijos y el poder doméstico para explicar la distribución N-S de la prevalencia de vida y uso actual de alcohol, tabaco y hoja de coca en el Perú, con excepción del uso actual de alcohol en la costa (León, 1987). La vitamina D tiene efectos importantes sobre neurotransmisores como la dopamina y la serotonina (Cass, Smith \& Peters, 2006) y es parte de la dinámica de la depresión estacional anual. Este síndrome consiste en la presencia recurrente de ciclos depresivos y se le atribuye a un déficit de exposición a rayos solares en el invierno; de allí que uno de sus tratamientos sea la terapia de luz (Rosenthal, Sack, Gillin, Lewy, Goodwin, Davenport et al., 1984). Relacionada a ella está la depresión invernal o nórdica, de alta incidencia en los países escandinavos, con la excepción de Islandia (Magnusson, Axelsson, Karlsson, Oskarsson \& Högni, 2000). 


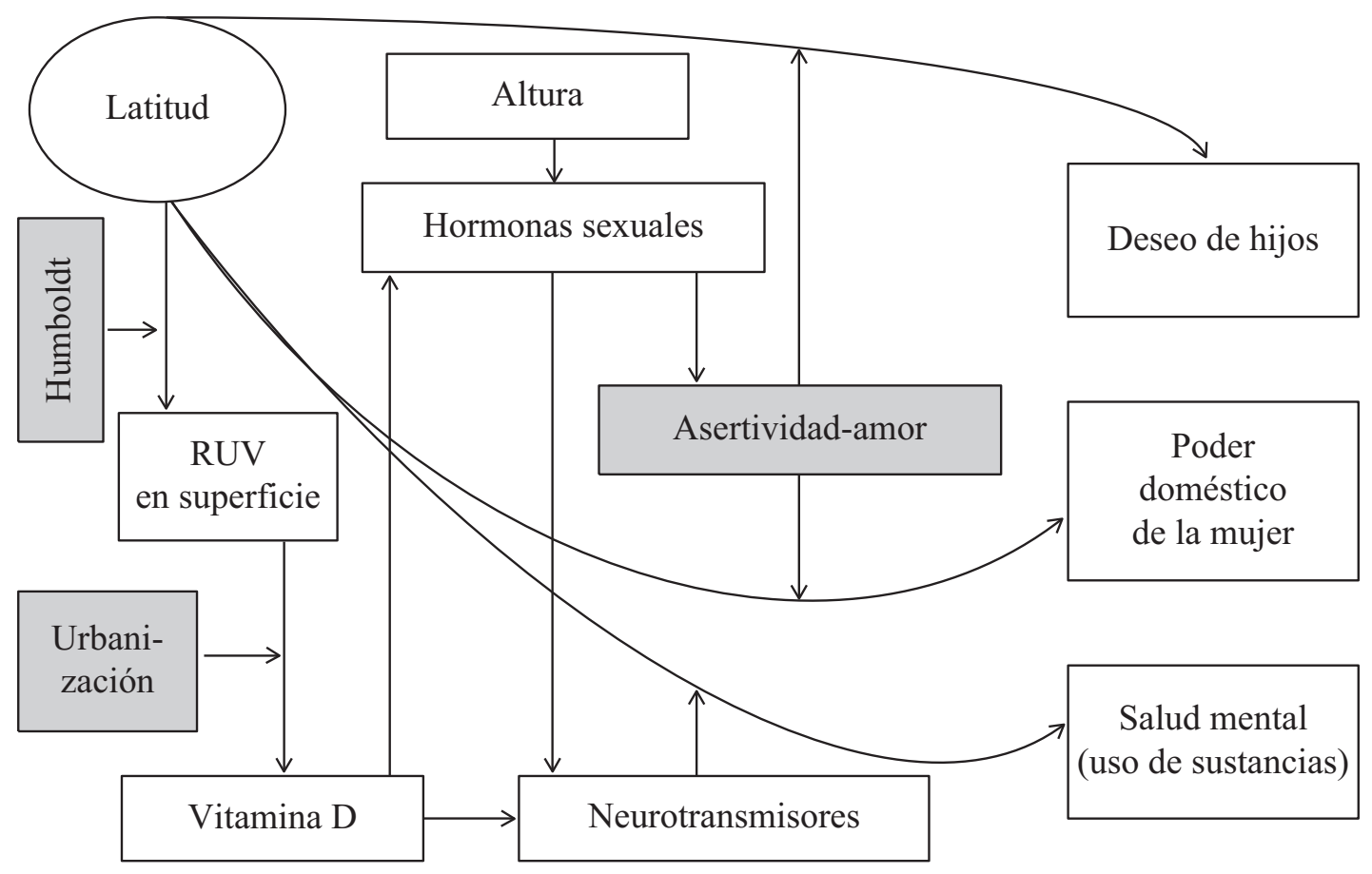

Figura 1. Teoría psicobiogeográfica del deseo de hijos, poder doméstico y salud mental: vínculos empíricos (líneas sólidas) y teóricos (guiones)

El déficit de RUV con la cercanía al Ártico da cuenta de la depresión nórdica, y la excepción se puede atribuir al muy alto consumo de pescado por parte de los islandeses (FAO, 2008); este alimento contiene importantes dosis de vitamina D. Finalmente, la testosterona, que teóricamente depende de la latitud, aumenta la producción de dopamina basal en hombres (Hull, Muschamps \& Sato, 2004), sugiriendo que sexualidad y estado de ánimo no son ajenos el uno al otro. El creciente uso de sustancias psicoactivas del N al S del Perú (León, 1987) puede, pues, entenderse como expresión de una necesidad de mejorar el estado de ánimo para contrarrestar los efectos depresivos de la menor intensidad de RUV sobre el cuerpo. Este mecanismo no contradice los efectos de la calidad de la relación madre-hijo en el $\mathrm{N}$ versus el $\mathrm{S}$ que imaginó León (1987) basado en una supuesta mayor autonomía y distancia emocional de las madres del S; a la distancia materna en el S se agregaría la comprometida salud mental de padres y madres sureños para aportar al consumo de sustancias psicoactivas por ellos mismos y por sus hijos. 
Violencia familiar peruana y teoría psicobiogeográfica / León

Otras variables consideradas son la corriente de Humboldt, la altura sobre el nivel del mar, y la dicotomía urbano-rural (León, 2012b). La teoría PBG predice que, a igualdad de otras condiciones, habrá menor deseo de hijos y salud mental y mayor poder doméstico de la mujer en la costa del Perú (debido a la niebla) que en otras regiones peruanas, excepto la sierra. En la sierra hay que considerar los efectos de la altura. La exposición a gran altura reduce la espermatogénesis (Gasco, Rubio, Chung, Villegas, \& Gonzales, 2003; Gonzales, 2007) y la testosterona en plasma (Gonzales, Kaneku \& Góńez, 1992). Similares efectos ocurren probablemente respecto a los niveles de estrógeno, pues la menarquia tiende a demorar (Gonzales \& Ortíz, 1994) y la menopausia a apresurarse (Gonzales \& Villena, 1997) en comparación con lo visto en la costa. Mientras que puede esperarse que la salud mental en la costa peruana, a causa de la corriente de Humboldt, sea peor que en la selva baja del Perú, no hay criterios a la mano para predecir un impacto mayor de la niebla costeńa versus la altura serrana en la salud mental. Finalmente, la teoría postula un efecto negativo de la urbanización sobre el deseo de hijos y la salud mental y positiva sobre el poder doméstico de la mujer, pues la vida urbana limita el acceso a RUV al asociarse con actividades bajo techo.

Dos reportes muy recientes prestan apoyo a la teoría PBG. El diario El Comercio (2012) informó que, de acuerdo a una investigación del Instituto Nacional de Salud Mental, las ciudades peruanas donde se encuentra mayor alegría son Tumbes, Tarapoto, y Chimbote, todas en el N, mientras que Ayacucho, Puno y Tacna, todas en el S, presentan los menores niveles de alegría; los investigadores atribuyeron las causas a factores genéticos y culturales. Blitchtein-Winicki y Reyes-Solari (2012) compararon la violencia de género contra la mujer, un indicador negativo de salud mental, en el territorio nacional. Se observa en sus datos que, excluyendo Lima Metropolitana (12\%), hay más violencia en el resto de la costa urbana (14.6\%) que en la rural (9.7\%), en la sierra urbana $(18.9 \%)$ que en la rural $(14.4 \%)$, y en la selva urbana $(17.8 \%)$ que en la rural $(16.3 \%)$. 
Puesto que los efectos de la latitud sobre el deseo de hijos y el poder doméstico de la mujer están plenamente demostrados en el Perú (León, 2011b, 2012a), el presente estudio puso a prueba la teoría PBG de la salud mental evaluando los efectos de la latitud sobre la violencia familiar. Además, evaluó los efectos de la altura, la urbanización, y la corriente de Humboldt sobre ella.

\section{Método}

El estudio cubrió las cinco ecorregiones Brack (1983) de orientación latitudinal.

\section{Ecorregiones del Perú}

El sistema geográfico de Brack (1983) aplica conceptos estándar de la literatura internacional, ofreciendo una descripción detallada de 11 ecorregiones (ver Figura 2). El sistema más reciente de Zamora (1996), de 18 ecorregiones, desagrega en exceso el territorio.

- (1) El desierto del Pacífico es de un ancho de 40 a $100 \mathrm{~km}$ y un rango altitudinal de 1 a 1000 metros sobre el nivel del mar (msnm); esta es una región altamente urbanizada e irrigada por cuatro decenas de ríos pequeños que bajan de los glaciares de los Andes y son alimentados por la lluvia serrana estacional. Corresponde a la región chala de Pulgar Vidal (1938). Esta es la ecorregión peruana que, en comparación con las siguientes cuatro, exhibe los mayores niveles de urbanización, densidad poblacional, riqueza del hogar, castellano como lengua materna, jefatura del hogar a cargo de una mujer, alfabetismo/educación, poder doméstico de la mujer, su edad, deseo de hijos, escucha de radio (empatado con la estepa), visión de TV, y lectura de periódicos, así como el menor número de hijos vivos (León, 2012b). 


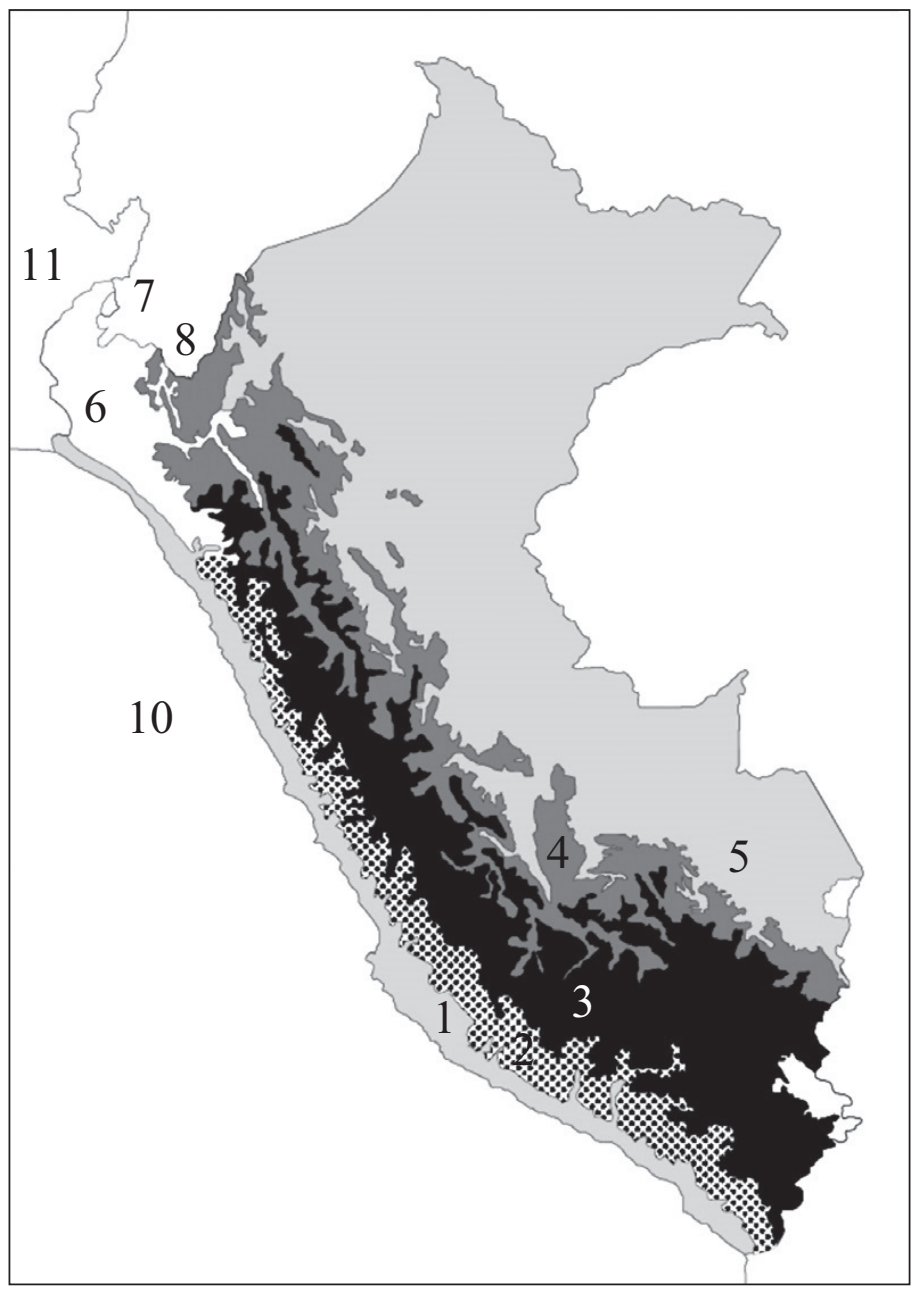

Figura 2. Las ecorregiones terrestres (1-9) y marítimas (10-11) del sistema geográfico de Brack (1983).

- (2) La estepa serrana es parcialmente equivalente a la región yunga marítima de Pulgar Vidal y los Andes meridionales de Zamora (1996); todos estos términos se refieren a los flancos occidentales de las montañas andinas (de 1000 a $3800 \mathrm{msnm}$ ). El valle de Chicama en el departamento de La Libertad representa la línea divisoria entre la estepa serrana y los flancos andinos más norteńos, clasificados por Brack como parte del bosque seco ecuatorial. La estepa se caracteriza por la mayor proporción de mujeres que trabajan, así como el menor tamaño del hogar. 
- (3) La puna de Brack no es la misma de Pulgar Vidal. Cubre territorios de gran altura desde el Parque Nacional de Cutervo (3200 msnm) en el sur del departamento de Cajamarca hasta los glaciares por encima de los 5200 msnm en el departamento de Tacna. Comprende varias de las regiones serranas de Pulgar Vidal y Zamora, incluyendo valles interandinos de menor altura donde se localizan ciudades como Cajamarca (2750 msnm) y Huaraz (2645 msnm). La puna se diferencia por su mayor número de niños vivos, así como menor urbanización, riqueza, alfabetismo/educación de la mujer, proporción que tiene al castellano como lengua materna, inmigración, diferencia de edad con el marido, deseo de hijos, ver TV, y leer periódicos.

- (4) La húmeda ecorregión yunga de Brack (la yunga fluvial de Pulgar Vidal) comprende los flancos orientales de los Andes, desde 600 a $3500 \mathrm{msnm}$ y es más caliente que la estepa serrana porque no está expuesta al frío traído por la corriente de Humboldt. La ecorregión yunga presenta el menor poder doméstico de la mujer (empatado con la Amazonía) y la menor proporción de mujeres como jefes del hogar.

- (5) La Amazonía peruana, desde los 50 a los $600 \mathrm{msnm}$, es conocida como selva baja. Su clima tropical es parecido al de África ecuatorial. Puede ser descrita como la ecorregión con las mujeres más jóvenes y mayor tamaño del hogar, nivel de inmigración, y diferencia de edad con el marido, así como menor densidad poblacional, mujeres que trabajan, poder doméstico de la mujer (empatado con la ecorregión yunga), y escucha de radio.

- Las otras ecorregiones son: el bosque seco ecuatorial (6), que ocupa la mayor parte de la costa norte y se extiende por el este desde Piura y Lambayaque hasta el río Marañón, cortando la ecorregión yunga en Cajamarca y haciéndole perder continuidad; el bosque húmedo tropical del Pacífico, que se encuentra en Tumbes (7); el páramo, en el N de Piura y Cajamarca (8); la sabana de palmeras, en Madre de Dios (9); el mar frío de la corriente de Humboldt (10); y el mar tropical (11). Solo las del 1 al 5, con un rango latitudinal mayor que 8o 50', fueron estudiadas por León $(2012 a, 2012 b)$ y lo son también en esta investigación. 
Violencia familiar peruana y teoría psicobiogeográfica / León

\section{Datos}

Fue necesario integrar cuatro bases de datos. El Geographically based Economy data (G-Econ 2011), un portal de internet desarrollado por William Nordhaus (Yale University) y Xi Chen (Quinnipiac University), proporcionó datos sobre densidad poblacional. Todas las otras variables del estudio provienen de bases de datos originadas en la Encuesta Demográfica y de Salud Familiar Perú 2000 (INEI \& Macro International, 2001). Se usó esta encuesta, y no la Continua 2004-2008, para aprovechar la construcción de variables geográficas de los estudios más recientes (León, 2012a, 2012b). La encuesta fue parte de la iniciativa internacional Demographic and Health Surveys (DHS), cuya metodología estándar a través de países implica un diseño de muestra multifásico y estratificado y pesos especialmente calculados para proporcionar estimados de las variables de interés con representatividad nacional y sub-nacional. Las fases del muestreo comprendieron la selección de conglomerados poblacionales y hogares dentro de ellos, la estratificación fue urbano-rural, y la representatividad alcanzó el nivel del departamento (ahora "región”). Entrevistas completas fueron obtenidas en el 88\% de las viviendas ocupadas. Los sujetos elegibles dentro de las viviendas eran todas las mujeres en edad fértil, es decir, de 15 a 49 años de edad; la tasa de respuesta individual fue de 95\%. Tres bases de datos de la encuesta - Geographic Positioning System (GPS), Wealth Index, e Individual Recode- fueron fusionadas. Ellas tenían, respectivamente, conglomerados $(\mathrm{N}=1409)$, viviendas $(\mathrm{N}=28$ 900), y mujeres $(\mathrm{N}=27$ 843) como casos.

\section{Variables independientes}

- Latitud absoluta. La base de datos GPS de DHS proporciona las coordenadas de latitud y longitud de cada conglomerado, levantadas a base del instrumento Garmin Etrex (Montana \& Spencer, 2005). La información se refiere al centroide del conglomerado y es presentada en grados decimales, no con los tradicionales minutos y segundos de la geografía. Se ignoró el signo y se asignó la misma latitud a todas las viviendas de un conglomerado y las mujeres dentro de esas viviendas. 
- Nivel latitudinal. Cada una de las ecorregiones del estudio con rango latitudinal mayor que $8^{\circ} 50^{\prime}$ fue dividida en tres partes iguales según los criterios mostrados en la Tabla 1. Las coordenadas de cada conglomerado de la encuesta fueron comparadas con una gigantografía del mapa de ecorregiones del Ministerio del Ambiente (2011) para decidir la clasificación de cada conglomerado en un nivel de latitud de una ecorregión o su exclusión del estudio. Los niveles de latitud (N, C, S) son iguales en extensión dentro de la ecorregión pero varían entre ecorregiones.

\section{Tabla 1}

Segmentación de cinco ecorregiones peruanas por nivel de latitud

\begin{tabular}{|c|c|c|c|c|c|}
\hline \multirow[b]{2}{*}{ Variables } & \multicolumn{5}{|c|}{ Ecorregiones } \\
\hline & Desierto & Estepa & Puna & Yunga & Amazonía \\
\hline $\begin{array}{l}\text { Grados límite } \\
\text { Norte }(\mathrm{N})\end{array}$ & $5,901 \mathrm{~S}$ & $7,901 \mathrm{~S}$ & $6,598 \mathrm{~S}$ & $5,095 \mathrm{~S}$ & $2,106 \mathrm{~S}$ \\
\hline $\begin{array}{l}\text { Grados límite } \\
\text { Centro (C) }\end{array}$ & $\begin{array}{l}10,001 \mathrm{~S} \\
14,100 \mathrm{~S}\end{array}$ & $\begin{array}{l}11,341 \mathrm{~S} \\
14,750 \mathrm{~S}\end{array}$ & $\begin{array}{l}10,228 \mathrm{~S} \\
13,857 \mathrm{~S}\end{array}$ & $\begin{array}{l}8,055 \mathrm{~S} \\
11,014 \mathrm{~S}\end{array}$ & $\begin{array}{l}5,776 \mathrm{~S} \\
9,445 \mathrm{~S}\end{array}$ \\
\hline $\begin{array}{l}\text { Grados límite } \\
\text { Sur }(S)\end{array}$ & $\begin{array}{l}14,101 \mathrm{~S} \\
18,190 \mathrm{~S}\end{array}$ & $\begin{array}{l}14,751 \mathrm{~S} \\
18,160 \mathrm{~S}\end{array}$ & $\begin{array}{l}13,858 \mathrm{~S} \\
17,487 \mathrm{~S}\end{array}$ & $\begin{array}{l}11,015 \mathrm{~S} \\
13,974 \mathrm{~S}\end{array}$ & $\begin{array}{l}9,446 \mathrm{~S} \\
13,115 \mathrm{~S}\end{array}$ \\
\hline $\begin{array}{l}\text { Ciudades } \\
\text { representativas } \\
\mathrm{N}\end{array}$ & $\begin{array}{l}\text { Chiclayo } \\
\text { s Trujillo } \\
\text { Chimbote } \\
\text { Huacho }\end{array}$ & $\begin{array}{l}\text { Otuzco } \\
\text { Caraz } \\
\text { Cajatambo } \\
\text { Canta } \\
\end{array}$ & $\begin{array}{l}\text { Cajamarca } \\
\text { Huamachuco } \\
\text { Huaraz } \\
\text { C. de Pasco }\end{array}$ & $\begin{array}{l}\text { Jaén } \\
\text { Moyobamba } \\
\text { Lamas } \\
\text { Uchiza }\end{array}$ & $\begin{array}{l}\text { Iquitos } \\
\text { S.M. de Nieva } \\
\text { Bagua } \\
\text { Yurimaguas }\end{array}$ \\
\hline $\begin{array}{l}\text { Ciudades } \\
\text { representa- } \\
\text { tivas C }\end{array}$ & $\begin{array}{l}\text { Lima } \\
\text { Ica } \\
\text { Nasca } \\
\text { Mollendo }\end{array}$ & $\begin{array}{l}\text { Yauyos } \\
\text { Huaytará } \\
\text { Arequipa } \\
\text { Moquegua }\end{array}$ & $\begin{array}{l}\text { Huancayo } \\
\text { Cusco } \\
\text { Sicuani } \\
\text { Azángaro }\end{array}$ & $\begin{array}{l}\text { Pozuzo } \\
\text { La Merced } \\
\text { Satipo } \\
\text { Quillabamba }\end{array}$ & $\begin{array}{l}\text { Juanjuí } \\
\text { Pucallpa } \\
\text { Atalaya } \\
\text { Manu }\end{array}$ \\
\hline $\begin{array}{l}\text { Ciudades } \\
\text { representa- } \\
\text { tivas } S\end{array}$ & Tacna & Tarata & Puno & Sandia & $\begin{array}{l}\text { Puerto } \\
\text { Maldonado }\end{array}$ \\
\hline $\begin{array}{l}\text { (Número } \\
\text { de casos } \mathrm{N} \text { ) }\end{array}$ & $(986)$ & $(221)$ & $(636)$ & $(1.064)$ & (589) \\
\hline $\begin{array}{l}\text { (Número } \\
\text { de casos C) }\end{array}$ & (1996) & $(81)$ & $(2261)$ & $(504)$ & (1043) \\
\hline $\begin{array}{l}\text { (Número } \\
\text { de casos } S \text { ) }\end{array}$ & $(785)$ & $(711)$ & $(1.266)$ & $(652)$ & $(789)$ \\
\hline
\end{tabular}


- Altura en msnm. Esta variable provino de la base de datos GPS. Se le dicotomizó en bajo $(<1000 \mathrm{msnm})=0$ y alto $(>999 \mathrm{msnm})=1$ para poder incorporarla en los gráficos junto con las dicotomías urbanorural y desierto-Amazonía. Los rangos efectivos respectivos fueron de 3 a 994 msnm y de 1000 a 4723 msnm. Los tamaños de muestra estuvieron bien balanceados: 6865 casos en tierras bajas y 7204 en la altura.

- Urbanización. DHS clasificó como urbano o rural el conglomerado al que pertenecía la vivienda de la mu-jer entrevistada. Esta clasificación respondió al criterio del INEI, según el cual un centro urbano tiene al menos 100 viviendas agrupadas contiguamente y/o es una capital distrital. Se recodificó rural $=1$ y urbano $=2$.

- Desierto-Amazonía. Esta variable es relevante a la prueba de los efectos de la corriente de Humboldt, vecina al desierto del Pacífico pero no de la Amazonía.

\section{Variable dependiente}

- Violencia familiar. Las opciones de respuesta para la pregunta "¿Alguna vez su esposo o compañero la ha empujado, golpeado, o agredido físicamente?” fueron recodificadas como No $=0$ y Sí $=1$. Se le preguntó a la mujer si vivía o había vivido las siguientes situaciones: $\mathrm{Su}$ esposo o compañero la ignora o es indiferente con Ud.; le prohíbe que estudie o trabaje; le impide ver a sus amistades o familiares; le grita cuando se dirige a Ud.; le dice "No sirves para nada" o frases similares; le dice "Me tienes cansado, me voy a ir de la casa"; le dice "Si te vas te quito mis hijos". Las respuestas fueron recodificadas Frecuentemente = 2 , A veces $=1, y$ Nunca $=0$. Se le preguntó también si su marido pasaba el tiempo libre con ella y si era carińoso con ella. Para estos ítems, las recodificaciones fueron Frecuentemente $=-2$, A veces $=-1, y$ Nunca $=$ 0 . El ítem "Hasta donde Ud. sabe, ¿su padre golpeaba a su madre?” dio lugar a las calificaciones 0 (No) y 1 (Sí). Cuando la mujer respondió Sí a la pregunta "¿Alguna persona (diferente a su esposo) la ha golpeado, abofeteado o le ha hecho algo que la ha herido a Ud. físicamente?" se indagó sobre la identidad del agresor. De esta manera, se pudo asociar 
puntajes 0 y 1 al padre y puntajes 0 y 1 a la madre. La suma de puntajes correspondientes a los 13 items de violencia familiar presentó una consistencia interna importante $(\alpha=.81)$. Solo 12604 casos ofrecieron respuestas a todos los ítems.

\section{Covariables}

- Densidad poblacional. Esta variable contextualiza al conglomerado. G-Econ (2011) proporciona

información poblacional por cada celda geográfica habitada de $1^{\circ}$ de latitud x $1^{\circ}$ de longitud en el mundo. En el caso del Perú, la información se refiere a 1990. La conversión de la información de base administrativa — datos censales de INEI (1991) por provincia- a celda geográfica la describen Deichmann, Balk y Yetman (2001). Las coordinadas de cada conglomerado fueron examinadas para decidir su correspondencia a una celda específica de G-Econ y se asignó a la mujer la población indicada para el conglomerado. Unos pocos conglomerados de Madre de Dios y Puno en la frontera con Bolivia carecían de información en la base de datos de G-Econ; en estos casos, se asignó la información poblacional existente para la celda peruana más cercana.

- Riqueza de la vivienda. DHS construye una variable de riqueza en cada país. Los ítems considerados incluyen la fuente de agua bebible (pozo, red pública, etcétera); tipo de instalaciones sanitarias (letrina, desagüe, etcétera); fuente de luz (eléctrica, etcétera); y material principal del piso, ventanas, y techo. Otros activos incluyen radio, televisión, refrigerador, bicicleta, motocicleta, y carro. Filmer y Pritchett (2001) describen el uso del análisis de componentes principales para obtener puntajes de riqueza a base de estos elementos. Implementan-do la metodología, DHS produce un puntaje estandarizado con media $=0 \mathrm{y}$ desviación estándar $=1$.

- Tamaño del hogar. El número total de miembros de la vivienda (adultos y niños) se obtuvo del Individual Recode.

- Etnia. Se le preguntó a la mujer, "¿Cuál es el idioma o lengua maternal que aprendió usted en su niñez?”. Las respuestas estaban 
codificadas en la base de datos como 1 = Castellano, 2 = Quechua, 3 = Aymara, 4 = Otra lengua aborígen, y $5=$ Idioma extranjero. Aquí fueron recodificados como Castellano $=1 \mathrm{y}$ cualquier lengua aborigen $=0$; idioma extranjero, que comprendía a menos del $0.1 \%$ de los casos, fue ignorado.

- Edad de la mujer. La edad de la mujer fue calculada considerando su fecha de nacimiento y la de la entrevista. DHS hace dos preguntas (“¿En qué mes y año nació usted?” y “¿Qué edad alcanzó en su último cumpleańos?") y corrige las inconsistencias en la medida de lo posible.

- Diferencia de edad con la del marido. DHS le preguntó a la mujer cual era la edad del marido. En el análisis de datos, se restó la edad de la mujer de la edad del marido.

- Oriunda del lugar. Se le preguntó a la mujer cuánto tiempo había vivido continuamente en el lugar de la entrevista. Las respuestas fueron recodificadas "Siempre" = 1 y "No siempre", "Inconsistente", o "No sé" $=0$.

- Jefe del hogar. Esta variable del Individual Recode fue recodificada Mujer $=1$, Hombre $=0$.

- Trabajo. Se le preguntó a la mujer si trabajaba al momento de la entrevista y si había trabajado en los últimos 12 meses. ("Como usted sabe, algunas mujeres trabajan por paga en dinero o especie. Otras venden cosas, tienen pequeńos negocios, o trabajan en la tierra o empresa de su familia. ¿Está usted haciendo actualmente alguno de estos trabajos? ¿Ha trabajado en los últimos 12 meses?”) Sus respuestas fueron recodificadas "No trabajó" = 0 y "Trabajó" y/o "Está trabajando actualmente" $=1$, independientemente de si recibía un pago y el tipo de pago.

- Número de hijos vivos. Esta es la suma de respuestas sobre el número de hijos varones y mujeres, sea que vivan con los padres o no.

- Alfabetismo/educación. Dos preguntas del cuestionario eran: “¿Alguna vez asistió a la escuela?” y “¿Cuál fue el año o grado de estudios más alto que aprobó?”. DHS calcula el número de ańos requerido para aprobar el grado más alto de estudios, incluyendo los de universidad. A las mujeres que dan una respuesta clasificable en "Instrucción primaria" o menos se les da una tarjeta con una oración y se les pide leerla. 
El estudio de León (2011b) trató independientemente a las dos variables, alfabetismo y educación. Aquí, como en León (2012a), se les combinó para producir la siguiente escala: $0=$ incapaz de leer; $1=$ lee parte de la oración y tiene $0-4$ ańos de escolaridad; 2 = lee correctamente y tiene 5-9 años de escolaridad; $3=10-11$ años; 4 = 12-14 años; $5=15-17$ años.

- Escucha de radio. DHS preguntó a la mujer si escuchaba radio y con qué frecuencia: nunca (0), menos de una vez a la semana (1), por lo menos una vez a la semana (2), o casi todos los días (3).

- Visión de TV. La pregunta y la codificación de esta variable son similares a las de "Escucha de radio".

- Lectura de periódicos. La pregunta y la codificación de esta variable son similares a las de "Escucha de radio".

\section{Enfoque analítico}

Como quiera que el diseño de la Encuesta Perú 2000 respondió al doble objetivo de derivar una muestra representativa a nivel nacional y 24 a nivel departamental, la muestra agregada a escala nacional sub-representó algunos departamentos y sobrerrepresentó otros. Por ejemplo, el tamaño de muestra de Lima $(\mathrm{N}=3714)$ estuvo por debajo de lo requerido por su importancia demográfica $(\mathrm{N}=9117)$ debido a que 3714 casos eran suficientes para conseguir un error estándar aceptable para los estimados departamentales; el de Madre de Dios $(\mathrm{N}=1418)$ fue mayor al que correspondía a su importancia poblacional $(\mathrm{N}=79)$ porque se habría podido hacer pocos estimados departamentales confiables sobre la base de 79 casos. DHS proporciona pesos muestrales para restablecer las proporciones adecuadas y obtener estimados representativos de las medias y porcentajes nacionales y departamentales. Sin embargo, las hipótesis del presente estudio no se refieren a medias o porcentajes sino a relaciones entre variables, y las ponderaciones de DHS no se aplican a correlaciones o regresiones (Rutstein \& Rojas, 2006). Se descartó la opción de ponderar los casos y obtener medias ponderadas para cada variable dependiente del estudio a cada nivel 
de latitud de cada ecorregión porque la única manera de evitar cualquier sesgo habría sido obtener $n$ muestras aleatorias de igual tamaño, una por grado de latitud $S$ por ecorregión; el uso de los pesos muestrales no compensaría por la falta de este diseño ideal. Por consiguiente, los análisis se ejecutaron sobre casos no ponderados. Se incluyó en los análisis solo a las mujeres cuyos esposos vivían con ellas.

El siguiente modelo lineal general se aplicó a la evaluación de los efectos del nivel de latitud:

$$
\mathrm{Y}=\mu+\delta+\left[\xi_{j}\right]+\varepsilon
$$

Aquí, Y es la medición de violencia doméstica, $\mu$ es su media poblacional, $\delta$ es el nivel de latitud

$(\mathrm{N}, \mathrm{C}, \mathrm{S}),\left[\xi_{j}\right]$ es un juego de covariables que incluye a la altura y urbanización, y $\varepsilon$ es el error aleatorio; es decir, estamos frente a un análisis univariado de la varianza de una vía que ańade el control sobre otras variables y permite graficar los resultados de la manera más efectiva. Una ecuación similar se aplicó en la evaluación de los efectos de la urbanización, la altura, y la corriente de Humboldt.

\section{Resultados}

La distribución de la suma de 13 ítems de violencia familiar a nivel nacional fue marcadamente sesgada (ver Figura 3). Nótese que el puntaje -4 se origina en la respuesta Frecuentemente ( -2 puntos) dada tanto al ítem sobre tiempo que comparte el esposo con la mujer como al de su comportamiento cariñoso con ella junto a respuestas 0 sobre violencia; el puntaje -3 indica una respuesta Frecuentemente ( -2 puntos) y una A veces (-1 punto) a los ítems sobre conducta benevolente del esposo y respuestas 0 sobre violencia; y así sucesivamente. El aspecto de distribución normal trunca se explica por la inclusión de solo dos ítems referidos a la benevolencia del esposo contra 11 pertinentes a violencia familiar. Descontando los dos ítems positivos se tendría la típica distribución J-invertida de las variables de desviación social. 


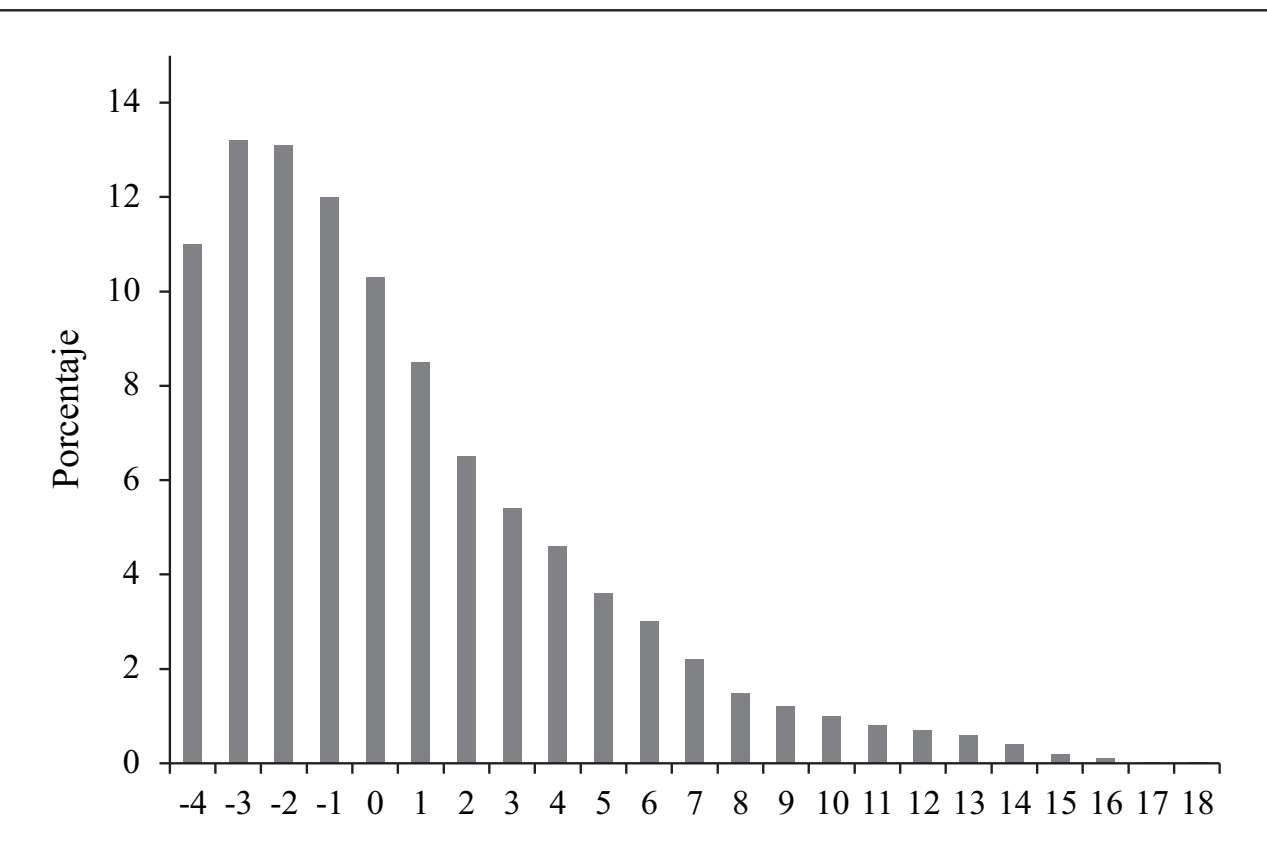

Figura 3. Distribución porcentual de frecuencias de la escala de violencia familiar en las cinco ecorregiones mayores del Perú

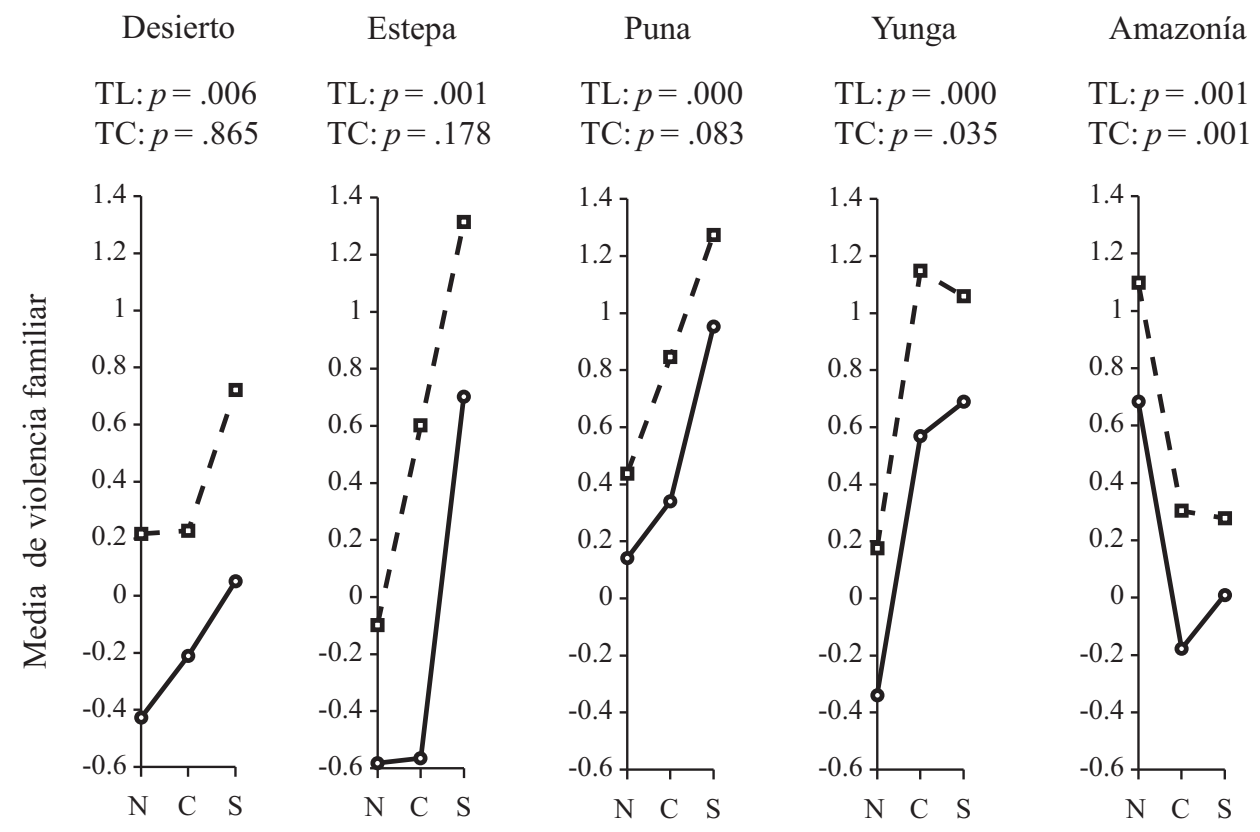

Figura 4. Medias de violencia familiar sin ajustar (línea intermitente) y ajustadas por 15 variables (línea sólida, según nivel de latitud (N, C, S) y ecorregión, y significación de las tendencias lineales (TL) y cuadráticas (TC) de las medias ajustadas 
Violencia familiar peruana y teoría psicobiogeográfica / León

\section{Tabla 2}

Tamaño de los efectos del nivel de latitud y otras 15 variables sobre la violencia familiar: valores eta ${ }^{2}$ parciales del modelo lineal general, por ecorregión

\begin{tabular}{|c|c|c|c|c|c|}
\hline \multirow[b]{2}{*}{ Variables } & \multicolumn{5}{|c|}{ Ecorregiones } \\
\hline & Desierto & Estepa & Puna & Yunga & Amazonía \\
\hline Nivel de latitud & $.002^{*}$ & $.014^{* * *}$ & $.007^{* * *}$ & $.016^{* * *}$ & $.010^{* * *}(-)$ \\
\hline Densidad poblacional & .000 & $.033^{* * *}$ & .001 & .001 & $.003^{*}$ \\
\hline Urbanización & $.004^{* * *}$ & .002 & $.007^{* * *}$ & $.003^{* *}$ & .001 \\
\hline Riqueza del hogar & $.003^{* *}(-)$ & $.009^{* *}(-)$ & $.004^{* * *}(-)$ & .001 & $.003^{*}(-)$ \\
\hline Número de residentes & .001 & .002 & .001 & $.003^{*}$ & .000 \\
\hline Etnicidad & .000 & .001 & .000 & $.003^{*}(-)$ & .000 \\
\hline Edad de la mujer & $.004^{* * *}$ & $.006^{*}$ & $.004^{* * *}$ & .000 & .000 \\
\hline Difer. con la del marido & .000 & $.010^{* *}$ & .000 & .000 & .000 \\
\hline Oriunda del lugar & $.003^{* * *}(-)$ & .002 & $.003^{* *}(-)$ & .002 & $.004^{* *}(-)$ \\
\hline Jefa del hogar & $.001^{*}$ & $.008^{* *}$ & .000 & .001 & .001 \\
\hline Trabajo & $.004^{* * *}$ & .000 & .000 & $.003^{* *}$ & $.002^{* *}$ \\
\hline Número de hijos & $.016^{* * *}$ & .000 & $.004^{* * *}$ & $.008^{* * *}$ & .000 \\
\hline Alfabetismo/educación & $.012^{* * *}(-)$ & $.014^{* * *}(-)$ & $.001^{*}(-)$ & $.006^{* * *}(-)$ & $.006^{* * *}(-)$ \\
\hline Escucha radio & .000 & .002 & .000 & .001 & .000 \\
\hline Ve TV & .001 & .000 & $.001^{*}(-)$ & .000 & .000 \\
\hline Lee periódicos & .001 & .000 & .000 & .001 & .000 \\
\hline $\mathrm{R}^{2}$ corregida & .085 & .105 & .046 & .059 & .045 \\
\hline$(\mathrm{N})$ & $(3521)$ & $(947)$ & $(3790)$ & $(2026)$ & $(2065)$ \\
\hline
\end{tabular}

Nota. El signo negativo junto a los eta ${ }^{2} s$ significativos indica que la violencia familiar decrecea con el incremento de la variable.

${ }^{+} p<.10, * p<.05, * * p<.01, * * * p<.001$. 
Se puede ver en la Figura 4 que la prueba de validez de la teoría PBG en lo referente a los efectos de la latitud arrojó evidencias confirmatorias en el desierto, la estepa, la puna, y la ecorregión yunga; en todas estas ecorregiones se observó un incremento significativo de la violencia familiar con el aumento de la latitud, independientemente de que los puntajes de violencia fueran brutos o ajustados por los efectos de las covariables. Sin embargo, resultados opuestos a la teoría emergieron en la Amazonía, donde la violencia familiar presentó puntajes significativamente más altos en el $\mathrm{N}$ que en el $\mathrm{C}(p<.000)$ o $\mathrm{S}(p<$ .001). El tamańo de los efectos se evaluó mediante eta ${ }^{2}$, obtenido en la ejecución del mismo modelo lineal general que produjo las medias ajustadas y las tendencias lineales y cuadráticas de la Figura 4. El efecto más consistente a través de regiones fue el del alfabetismo/educación de la mujer, correlacionado negativamente con la violencia familiar. Les siguieron los de la latitud y la riqueza del hogar (esta con signo negativo). También presentaron cierta consistencia interregional los efectos de la urbanización, edad de la mujer, migración (inverso de oriunda del lugar), trabajo, y número de hijos.

Sin ajuste

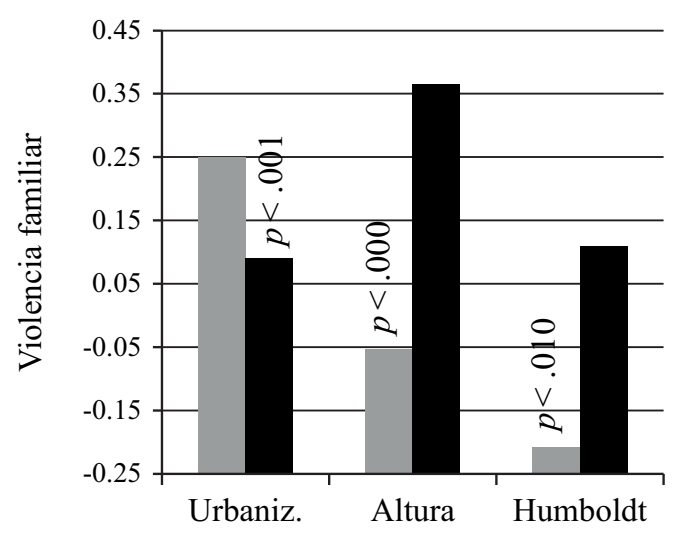

Con ajuste

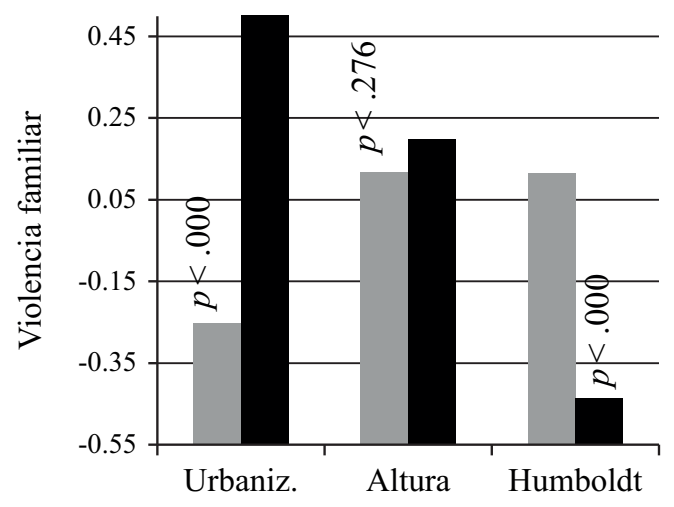

Figura 5. Medias de violencia familiar sin ajustar y ajustadas por 16 covariables en ámbito rural (gris) o urbano (negro), tierras bajas (gris) o tierras altas (negro) y desierto del Pacífico (gris) o Amazonía (negro) y significación de las diferencias 
Las cinco ecorregiones del estudio fueron integradas para las pruebas de validez de las siguientes hipótesis. La Figura 5 presenta resultados relevantes a la evaluación de los efectos de la urbanización, la altura, y la corriente de Humboldt sobre la violencia familiar. Los puntajes sin ajuste de violencia familiar contradijeron a la teoría al mostrar significativamente mayor violencia en espacios rurales que en áreas urbanas y en la Amazonía que en desierto del Pacífico. Solo los efectos de la altura respaldaron a la teoría. Sin embargo, la teoría no se refiere a las cosas tal como son sino a cómo serían si se pudiera aislar los efectos de la latitud, urbanización, altura, y corriente de Humboldt de los efectos de otras variables sobre la violencia familiar. Pese a recibir mayor RUV, la violencia es mayor en ámbitos rurales a causa de la mayor pobreza, menor educación, etc., y lo mismo vale para la Amazonía. Cuando los puntajes fueron ajustados se invirtieron las relaciones para urbanización y la dicotomía desierto-Amazonía (Humboldt), aunque perdió significación el efecto de la altura. Los ajustes fueron hechos respecto a las mismas covariables de la Tabla 2, más la latitud absoluta.

\section{Comentarios}

La teoría PBG sale fortalecida de este estudio con la demostración de que la violencia familiar crece de $\mathrm{N}$ a $\mathrm{S}$ en la mayor parte del territorio peruano, pues los resultados complementan hallazgos relevantes del pasado que se referían a los efectos de la latitud en otra área de la salud mental, la del uso de sustancias psicoactivas (León, 1987), o eran asistemáticos al ignorar la variable latitudinal per se (El Comercio, 2012). Ahora es posible afirmar que, de acuerdo con lo predicho por la teoría, la salud mental, un constructo de mayor jerarquía, que abarca tanto el uso de sustancias psicoactivas como la violencia familiar, se deteriora sistemáticamente de $\mathrm{N}$ a $\mathrm{S}$ en la mayor parte del territorio nacional. La excepción estuvo dada por la Amazonía. 
¿Por qué no funcionó allí la teoría? ¿Por qué sí funcionó en el estudio previo (León 2012a) respecto al deseo de hijos y el poder doméstico de la mujer? Estas variables presentaron tendencias lineales significativas en las direcciones esperadas desde el $\mathrm{N}$ hasta el $\mathrm{S}$ de la Amazonía (definidos como aquí).Una posibilidad es que sea lineal la relación entre el nivel de vitamina $\mathrm{D}$ en plasma y el de las hormonas sexuales que incentivan el deseo de hijos e inhiben el poder doméstico de la mujer, pero sea curvilineal la relación entre el nivel de vitamina D y el de neurotransmisores como la dopamina y la serotonina. Tal vez haya un límite a partir del cual se revierte el signo de la relación. Se puede notar en la Figura 2 y la Tabla 1 que el nivel $\mathrm{N}$ de la Amazonía está totalmente más al $\mathrm{N}$ que el $\mathrm{N}$ del desierto, la estepa, o la puna $\mathrm{y}$ parcialmente más al $\mathrm{N}$ que el $\mathrm{N}$ de la ecorregión yunga. Tal vez las relaciones postuladas por la teoría entre la latitud y la violencia doméstica sean lineales aproximadamente desde la latitud 50 50' $\mathrm{S}$ hasta los bordes sureños del país, pero sufran una inflexión desde la latitud 50 50' $\mathrm{S}$ hacia el $\mathrm{N}$ si el nivel de vitamina $\mathrm{D}$ producido a esas latitudes deviene en excesivo para los procesos de neurotransmisión.

La tendencia cuadrática significativa observada en la Amazonía, que expresa la caída de la violencia familiar de $\mathrm{N}$ a C y su elevación de $\mathrm{C}$ a $\mathrm{S}$ vistos en la Figura 4, es consistente con esta idea. Para verificarla sería necesario practicar observaciones en espacios habitados desde la línea ecuatorial hasta espacios cercanos a los trópicos de Cáncer y Capricornio. Por ejemplo, desde la latitud $0^{\circ} 0^{\prime}$ en Kenya hasta el borde $\mathrm{N}$ de Etiopía y $\mathrm{S}$ de Tanzania. Un primer requerimiento de validez sería la replicación de los hallazgos peruanos, es decir, la presencia de una relación lineal de la latitud con el deseo de hijos y el poder doméstico (León 2012a) y curvilinear con la violencia familiar (este estudio). En un contexto geográfico amplio, también habría que abrirse a la posibilidad de otras curvilinealidades. Por ejemplo, parece improbable que la violencia familiar siga aumentando ilimitadamente hacia el $S$, pues ello implicaría que debe esperarse ver maridos más violentos con sus parejas en Buenos Aires que en Puno, lo cual contradeciría importantes estereotipos sociales. 
Los estereotipos sociales son políticamente incorrectos, pero no se puede ignorar a rajatabla que algo de verdad tiene que haber en sus orígenes. ¿O se expresará el deterioro de la salud mental como violencia familiar en el tramo inicial de la latitud $S$ (por ejemplo, de Ecuador a Bolivia) y más en la forma de depresión clínica en el siguiente tramo (hasta la Patagonia)? Cualquiera de estas opciones tendría su contraparte en el hemisferio $\mathrm{N}$, por supuesto. Otra posibilidad es que a partir de cierto nivel de RUV se presenten interacciones entre variables dependientes de la teoría. Por ejemplo, el incremento multiplicado del interés sexual de la mujer con la cercanía a la línea ecuatorial, algo que es consistente con el estereotipo del "ardor sexual de la mujer selvática" en el Perú, podría causar respuestas violentas del marido ante sospechas de infidelidad.

De las otras hipótesis que fueron evaluadas, las referidas a la urbanización y la corriente de Humboldt condujeron a los hallazgos más consistentes con la teoría PBG. Los puntajes ajustados de violencia familiar presentaron mayores niveles en ámbitos urbanos que rurales y en el desierto del Pacífico que en la Amazonía. También presentaron la dirección esperada en el contraste entre tierras bajas y altas, pero sin alcanzar significación estadística. La diferencia con los otros dos casos puede entenderse considerando la parsimonia o fortaleza teórica de los postulados que involucran a la urbanización y la corriente de Humboldt versus aquellos asociados con los efectos de la altura. La teoría PBG postula que la violencia familiar será mayor en ámbitos donde la humanidad está menos expuesta a RUV dadas sus actividades bajo techo o estar sometidos a la bruma. Ello implica asumir un supuesto simple y ya demostrado científicamente: la vitamina D tiene efectos significativos sobre neurotransmisores relevantes a la salud mental. En cambio, el que la altura, al disminuir el nivel de las hormonas sexuales, induzca mayor violencia familiar requiere el supuesto adicional de una relación entre sexualidad y salud mental que podría ser menos fuerte o podría estar moderada por contingencias desconocidas. 
En conclusión, los hallazgos sugieren que el componente latitudinal de la teoría PBG sobre la salud mental tiene una validez considerable pese a la excepción observada en la Amazonía. Las nuevas hipótesis planteadas para explicar los resultados amazónicos podrían enriquecer substancialmente a la teoría una vez que sean sometidas a prueba. Los efectos de la urbanización y la corriente de Humboldt también se comportaron de acuerdo a lo predicho por la teoría. Es verdad que hubo limitaciones metodológicas: puesto que las muestras no fueron estrictamente representativas de los grados de latitud, sesgos desconocidos podrían haber afectado las relaciones observadas. Aparte de partir de un diseño desbalanceado (véase la Tabla 1), se violó el supuesto de distribución normal de la variable dependiente en el análisis de la varianza; además, la presencia de errores correlacionados espacialmente podría haber causado sobreestimados de los impactos. Pero el análisis de la varianza es una técnica robusta que suelen trascender la violación de sus supuestos y la consistencia de los hallazgos con los de otras investigaciones (e.g., Blitchtein-Zinicki \& Reyes-Solari, 2012; El Comercio, 2012) sugieren que estamos ante evidencias replicables. En cualquier caso, si aparecieran en algún momento hipótesis competidoras que generaran evidencias capaces de desplazar parcial o enteramente a la teoría PBG, ello solo significaría una demostración de vigor de la psicología peruana.

En cambio, parece necesario revaluar las hipótesis sobre la altura. Esto puede hacerse refinando las hipótesis y/o fortaleciendo la metodología. Lo primero demanda imaginación científica. Lo segundo parece ser más asequible, al menos en teoría: es evidente que un mejor muestreo, la medición directa de RUV, y el recurso a ecuaciones estructurales fortalecerían la línea de investigación presentada en este artículo. 


\section{Referencias}

Bem, S. L. (1974). The measurement of psychological androgyny. Journal of Consulting and Clinical Psychology, 42, 115-162.

Blitchtein-Winicki, D. \& Reyes-Solari, E. (2012). Factores asociados a violencia física reciente de pareja hacia la mujer en el Perú, 2004-2007. Revista Peruana de Medicina Experimental y Salud Pública, 29, 35-43.

Brack, A. (1983). El ambiente en que vivimos. Lima: Editorial Salesiana.

Cass, W. A., Smith, M. P., \& Peters, L. E. (2006). Calcitriol protects against the dopamine- and serotonin-depleting effects of neurotoxic doses of methanphetamine. Annals of the New York Academy of Sciences, 1074, 261-271.

Costa Jr., P. T., \& McCrae, R. R. (1992). Revised NEO Personality Inventory (NEO-PI-R) and NEO Five Factor Inventory (NEO-FFI) professional manual. Odessa, FL: Psychological Assessment Resources.

Costa Jr., P. T., Terracciano, A., \& McCrae, R. R. (2001). Gender differences in personality traits across cultures: robust and surprising findings. Journal of Personalityy and Social Psychology 81, 322-331.

Cummings, D. R. (2007). Additional confirmation for the effects of environmental light intensity on the seasonality of human conceptions. Journal of Biosocial Science, 39(3), 383-396.

Deichman, U., Balk, D. \& Yetman, G. (2001). Transforming population data for interdisciplinary usages: from census to grid. Recuperado de http://sedac.ciesin.columbia.edu/plue/gpw/ index.html?grl.html \&2

El Comercio (2012). Los peruanos más alegres están en Chimbote, Tarapoto, y Tumbes. Recuperado de http://elcomercio.pe/ peru/1419844/noticia-peruanos-mas-alegres-estan-chimbotetarapoto-tumbes

Eysenck, H. J. \& Eysenck, M. W. (1985). Personality and individual differences: A natural science approach. Nueva York: Plenum. 
FAO (2008). Fishery and aquaculture statistics: Section 2. Food balance sheets and fish contribution to protein supply, by country from 1961 to 2007. Roma: Food and Agriculture Organization.

Filmer, D., \& Pritchett, L. H. (2001). Estimating wealth effects without expenditure data - or tears: an application to educational enrollments in states of India. Demography, 38, 1, 115-132.

Gasco, M., Rubio, J., Chung, A., Villegas, L. \& Gonzales G. F. (2003). Effect of high altitude exposure on spermatogenesis and epididymal sperm count in male rats. Andrologia, 35, 368-374.

G-Econ. (2011). Geographically Based Economic Data. Recuperado de http://gecon.yale.edu/.

Gonzales, G. F. (2007). Peruvian contributions to the study on human reproduction at high altitude: from the chronicles of the Spanish conquest to the present. Respiratory Physiology \& Neurobiology, 158, 172-176.

Gonzales, G. F., Kaneku, L. \& Góñez, C. (1992). Fisiología y patología de la reproducción en las grandes alturas. Acta Andina, 1, 3-16.

Gonzales, G. F. \& Ortíz, I. (1994). Age at menarche at sea level and high altitude in Peruvian women of different ethnic background. American Journal of Human Biology, 6, 674-640.

Gonzales, G. F. \& Villena, A. (1997). Age at menopause in central Andean Peruvian women. Menopause, 4, 32-38.

Goldberg, L. R. (1973). The structure of phenotipic personality traits. American Psychologist, 48, 26-34.

Hull, E. M., Muschamps, J. W. \& Sato, S. (2004) Dopamine and serotonin: influences on male sexual behavior. Physiology \& Behavior, 83, 2, 291-307.

Huhtainemi, I., Martikainen, H. \& Tepanainen, J. (1982). Large annual variation in photoperiodicity does not affect testicular endocrine function in men. Acta Endocrinologica, 101, 105-107.

INEI (1991). Censos Nacionales 1990. Lima: Instituto Nacional de Estadística e Informática. 
INEI \& Macro Inernational (2001). Perú 2000: Encuesta Demográfica $y$ de Salud Familiar. Lima: Instituto Nacional de Estadística e Informática.

Kabeer, N. (2001). Reflections on the measurement of empowerment. En N. Kabeer, P. McFadden, S. Arnfred, E. Dominguez \& S. Sudallaah (Eds.), Discussing women's empowerment - Theory and practice, pp. 17-57. Stockholm: Novum Grafiska AB, SIDA Studies No. 3.

Kauffmann-Doig, F. (1979). Sexual behavior in ancient Peru. Lima: Kompaktos.

Kinuta, K., Tanaka, H., Moriwake, T., Aya, K., Kato, S. \& Seino, Y. (2000). Vitamin D is an important factor in estrogen biosynthesis of both female and male gonads. Endocrinology, 141, 4, 1317-1324.

Kishor, S. \& Subaiya, L. (2008). Understanding women's empowerment: A comparative analysis of Demographic and Health Surveys data. DHS Comparative Studies No. 20.

León, F. R. (1984). El eje fecundatorio norte-sur del Perú: Una interpretación psicológica. Revista de Psicología, 2, 95-111.

León, F. R. (1986). Factores psicosociales, psicoeconómicos, y psicosexuales en el eje fecundatorio norte-sur del Perú. En F. R. León (Ed.), Psicologia y realidad peruana: El aporte objetivo. Lima: Mosca Azul Editores.

León, F. R. (1987). El eje psicoactivo norte-sur del Perú. Psicoactiva, 1, 3-13.

León, F. R. (2011a). Does professed religion moderate the relationship between women's domestic power and contraceptive use in India? Open Family Studies Journal 4, 1-8.

León, F. R. (2011b). Latitud sur y control económico del hogar por la mujer peruana. Revista de Psicología, 29, 2, 361-388.

León, F. R. (2012a). Una teoría psicobiogeográfica del poder doméstico de la mujer. Revista Peruana de Psicología y Trabajo Social, $1,29-44$. 
León, F. R. (2012b). The latitudinal tilts of wealth and education in Peru: Testing them, explaining them, and reflecting on them. Economia, en prensa.

MacLean, C. P. \& Anderson, E. R. (2009). Brave men and timid women? A review of the gender differences in fear and anxiety. Clinical Psychology Review, 29, 6, 496-505.

Magnusson, A., Axelsson, J., Karlsson, M. M., Oskarsson, H. (2000). Lack of seasonal mood change in the Icelandic population: results of a cross-sectional study. American Journal of Psychiatry, 157, 2, 234-238.

Malhotra, A., Schuler, S. R. \& Boender, C. (2002). Measuring women's empowerment as a variable in international development. Washington, DC: World Bank's Social Development Group.

Mason, K. O. (1998). Gender and Family systems in the fertility transition. Population and Development Review, 27, 160-176.

Ministerio del Ambiente del Perú (2011). http://geoservidor.minam.gob. pe/atlasperu/son/001.mp3.

Montana, L. \& Spencer, J. (2005). Incorporating geographic information into MEASURE surveys: a field guide to GPS data collection. Chapel Hill: Carolina Population Center.

Morales, T. (1983). Intenciones reproductivas. En: Instituto Nacional de Estadística, Aspectos demográficos y prevalencia de anticonceptivos: Resultados de la Primera Encuesta Nacional de Prevalencia de Anticonceptivos. Lima: Autor.

Pulgar Vidal, J. (1938). Las ocho regiones naturales del Perú. Lima: Universidad Nacional Mayor de San Marcos, Tesis.

Rosenthal, N. E., Sack, D. A., Gillin, J. C., Lewy, A. J., Goodwin, K., Davenport, Y., Mueller, P. S., Newsome, D. A., Weher, T.A. et al. (1984). Seasonal affective disorder: a description of the syndrome and preliminary findings with light therapy. Archives of General Psychiatry, 41, 1, 72-80.

Rutstein, S. O. \& Rojas, H. (2006). Guide to DHS statistics. Calverton, MD: Demographic and Health Surveys, ORC Macro. 
Sen, A. (1999). Development as freedom. Londres: Oxford University Press.

TuTiempo.net (2012). Recuperado de html://www.tiempo.net/tiempo/. van Anders, S. M., Hampson, E. \& Watson, N. V. (2006). Seasonality, waist-to-hip ratio, and salivary testosterone. Psychoneuroendocrinology, 31, 7, 895-899.

Wehr, E., Pitz, S., Boehm, B. O., März, W. \& Obermayer-Pietsch, B. (2009). Association of vitamin D status with serum androgen levels in men. Clinical Endocrinology, 73, 2, 243-248.

Wiggins, J. S. (1979). A psychological taxonomy of trait descriptive terms: The interpersonal domain. Journal of Personality and Social Psychology, 37, 395-412.

Zamora, C. (1996). Las regiones ecológicas del Perú. En Lily O. Rodríguez (Ed.), Diversidad biológica del Perú: zonas prioritarias para su conservación. Lima: Proyecto Fanpe GTZ-INRENA.

Recibido: 20 de junio de 2012 Aceptado: 17 de septiembre de 2012 